\title{
Application and Efficacy of Pubovesical Complex Preserving Technique During Laparoscopic Radical Prostatectomy
}

\author{
Chen Jiajun, Zhu Zaisheng*, Shi Hongqi, Zhou Pengfei, Xu Lizhen, Zhou Yibo, Zhu Yiyi \\ Department of Urology, Jinhua Hospital, Zhejiang University School of Medicine, Jinhua, China
}

Email address:

zaisheng_zhu@163.com (Zhu Zaisheng)

${ }^{*}$ Corresponding author

\section{To cite this article:}

Chen Jiajun, Zhu Zaisheng, Shi Hongqi, Zhou Pengfei, Xu Lizhen, Zhou Yibo, Zhu Yiyi. Application and Efficacy of Pubovesical Complex Preserving Technique During Laparoscopic Radical Prostatectomy. Journal of Surger. Vol. 7, No. 3, 2019, pp. 67-73.

doi: $10.11648 /$ j.js.20190703.14

Received: December 10, 2018; Accepted: June 12, 2019; Published: June 15, 2019

\begin{abstract}
Urinary incontinence and Erectile function are two hot issues after radical prostatectomy. This study aims to explore the application and curative effect of "pubovesical complex preserving technique for intrafascial laparoscopic radical prostatectomy (PPLRP)". Methods: This was a retrospective study of 200 patients who underwent laparoscopic radical prostatectomy (LRP) from January 2011 to May 2018. Results: During the study period, we collected 68 patients with PPLRP in group A, 46 patients with conventional intrafascial LRP in group B and 86 patients with interfascial LRP in group C, respectively. Three groups had no difference in terms of Preoperative age, Clinical stage, Prostate-specific antigen (PSA) value, Gleason score at biopsy and Preoperative good potency (IIEF-5 score) $(\mathrm{P}>0.05)$. There was no difference between the three groups in terms of operative time, intraoperative blood loss, time of catheter retain, postoperative stay and histologic status. All groups had similar PSM (positive surgical margin, PSM) rate. Continence rates at immediately after catheter removal, 1,3 and 6 months: $68 \%(46 / 68), 85 \%(58 / 68), 93 \%$ (63/68) and 100\% (68/68) in group A; and 63\% (29/46),80\% (37/46),89\% (41/46) and $96 \%(44 / 46)$ in group B; and 22\% (19/63),49\% (42/63),72\% (62/63) and $84 \%(72 / 63)$ in group C. Regarding sexual function: No differences were found in the three groups in potency (IIEF-5 score). Baseline IIEF-score reached 50\%,35\% and $21 \%$ respectively at postoperative 6 months. Biochemical recurrence-free survival at 3 years was $79.3 \%, 76.3 \%$ and $76.4 \%$ in A, $\mathrm{B}$ and $\mathrm{C}$ group, respectively. Conclusions: The PVC preserving technique for LRP provides early recovery from incontinence, faster recovery of sexual function preoperative levels. There is no adverse effect on the oncological outcome in the selective prostate cancer. It is an ideal way of nerve-sparing LRP.
\end{abstract}

Keywords: Laparoscopic Surgery, Prostate Cancer, Intrafascial Nerve-Sparing, Interfascial Nerve-Sparing, Erectile Function, Urinary Incontinence

\section{Introduction}

The main complications of LRP are urinary incontinence and Erectile dysfunction, especially in the early postoperative period $[1,2]$. Recently there have been more reports about intrafascial LRP [3, 4] to reduce these complications. LRP provides precise surgical procedures, because it allows for clear, highly magnified visualization, which has led to a better microscopic anatomy around the prostate. At the same time, it also has the advantage of less intraoperative bleeding. In July 2012, we started using a surgical procedure called PPLEP. We retain all tissue structures around the prostate, including the Denonvillier ligament, the detrusor apron, the Dorsal vein complex (DVC) and the Neurovascular bundle (NVB). This technique preserves the nerves and blood vessels around the prostate and urethra more widely than traditional intrafascial nerve retention LRP. This technique also has satisfactory results in oncology and functional recovery. 


\section{Method}

\subsection{General Information}

From January 2011 to May 2018, a retrospective study of 200 patients who underwent LRP. Mean age were 63.3 (46 75) years, Course of disease $4.2(0.25 \sim 14.0)$ months. Clinical manifestations: 33 cases of dysuria, hematuria in 12 cases, 38 cases of urinary frequency and 119 cases of asymptomatic. TNM clinical stages [5]: 136 cases of $\mathrm{cT}_{1}$ stage and 64 cases of $\mathrm{cT}_{2}$ stage. Patient with hypertension 43 cases, 20 cases of diabetes, 7 cases of coronary heart disease and 4 cases of cerebral infarction. clinical examination and laboratory examinations: Preoperative PSA 12.0 (3.4 34.7) $\mu \mathrm{g} / \mathrm{ml}$, The volume of the prostate was measured by transrectal ultrasound before surgery, 45.3 (18.4-121.9) $\mathrm{ml}$. All patients underwent radionuclide bone scan to exclude bone metastases; Neither MRI nor CT showed significant prostatic extracapsular invasion and lymph node metastasis. All patients underwent $8+x$ transperineal prostate biopsy guided by transrectal ultrasound before operation. The distribution of the 8 needles was 2 needles on the left and right sides of the external gland, 1 needles on the left and right sides of the junction of the internal and external glands, and 1 needles on the left and right sides of the internal gland. Puncture X needles in suspicious areas (prostatic nodule). All patients were diagnosed as prostate cancer by puncture pathology. The Gleason score of biopsy was 5-9 points with an average of 6.7 points. Patients' erectile function were evaluated with the International Index of Erectile Function (IIEF-5) [4, 5]: The Preoperative IIEF-5 score $\geq 15$ was considered to have better erectile function. Confounding factors were excluded: TNM clinical stage $>$ cT3, lack of important clinical data such as pathology report, preoperative neoadjuvant endocrine therapy or radiotherapy, postoperative follow-up $<6$ months and patients with excessive PSA.

Of the 200 patients, 68 patients (group A) underwent PPLRP technique, which did not cut pelvic fascia and suture deep vein complex. The periprostatic anatomy was preserved by keeping the pubovesical complex (PVC), including detrusor apron, pubovesical ligaments, DVC and NVB;46 patients (group B) underwent traditional intrafascial technique and 86 patients (group C) underwent interfascial technique. The general data of the three groups were compared in Table 1. There was no significant difference in terms of preoperative age, clinical stage, PSA value, Gleason score at biopsy and prostate volume $(\mathrm{P}>0.05)$.

\subsection{The Surgical Method}

After tracheal intubation combined with intravenous anesthesia, the patient took a low foot height of $15^{\circ} \sim 20^{\circ}$. Make a longitudinal incision about $2.5 \mathrm{~cm}$ under the umbilicus. Separate into the abdominal wall and bluntly separate the extraperitoneal space. $10 \mathrm{~mm}$ Trocar (Right) and $5 \mathrm{~mm}$ Trocar (Left) were punctured on the lateral border of rectus abdominis muscle, $2.0 \mathrm{~cm}$ below the incision. Put $10 \mathrm{~mm}$ Trocars in the incision, sutured and connected laparoscopic pneumoperitoneum. Maintain extraperitoneal pressure to $12-15 \mathrm{mmHg}(1 \mathrm{mmHg}=0.133 \mathrm{kPa})$. Two $5 \mathrm{~mm}$ Trocars were punctured on $3 \mathrm{~cm}$ above the bilateral superior anterior superior iliac spine under direct vision (4-hole surgery started in 2015).

Identify the bladder neck $(\mathrm{BN})$ and the bottom of the prostate. Then at the junction of the $\mathrm{BN}$ and prostate of 11 o'clock 1 o'clock, make a transverse incision of about $2 \mathrm{~cm}$ in the BN. From the incision, bluntly separate the BN. BN was found from both sides using the $\mathrm{BN}$ preservation technique, exposing the junction of the urethra and the BN. Incision the $\mathrm{BN}$ until the catheter was seen. Pull the catheter to the pubic symphysis to pull the prostate and cut the posterior portion of the BN. Identify the seminal vesicle and the ampulla ductus deferentis, cut the bilateral vas deferens near the bottom of the seminal vesicle, cut off the seminal vesicle arteries, and peel the dense Denonvilliers fascia close to the seminiferous glands to the plane of the ejaculatory duct, and transverse cut the Denonvilliers fascia and keep it. On the side where the nerve is retained, blunt dissection the prostate capsule and the fascia, and the inner layer of the fascia is easily peeled off. The side of the prostate is separated bluntly or sharply using an ultrasonic scalpel or scissors, from posterior to forward to the prostatic tip and lateral anterior surface (Figure 1A). If necessary, use a titanium clip or Hem-o-lok to clamp the vascular pedicle of the prostate, and avoid damaging NVB. DVC was isolated from the fibrous interstitium of anterior prostatic muscle and the prostate. Non-suture the DVC (in some cases, the DVC was sutured in the medial side), Close to the prostate capsule and annularly separate the prostatic fascia to the urethra (Figure 1B). Remove the fascia from the urethra and cut the entire urethra horizontally with scissors (Figure 1C). Completely preserved DVC, detrusor apron and puboprostatic ligament, the structures surround the pelvic floor, apex prostatic and urethra including nerves and blood vessels (such as NVB, DVC and accessory pudendal artery) are widely preserved (Figure 1D). Reconstruct the posterior side of the BN, use the 2-0 absorbable suture, continuous suture bladder and urethra, knotted on the outside (Figure 1E). Reconstruct the PVC (Figure 1F). Indwelling pelvic drainage tube.

\subsection{Postoperative Management and Effectiveness Evaluation}

The perioperative conditions of the 3 groups were compared: operative time, intraoperative blood loss (including intraoperative urine volume), complications and postoperative hospital stay, etc., postoperative pathological stage (pT), Gleason score, PSM rate, etc.

Anti-infection treatment was routinely used for 1 weeks after operation, the pelvic drainage tube was removed 3 to 5 days after operation, and the catheter was removed 10 to 14 days later. Endocrine therapy with total androgen blockade was performed on patients with PSM for 6 months (Leuprorelin Acetate Microspheres for Injection 3. $75 \mathrm{mg}$ or Goserelin acetate $3.6 \mathrm{mg}$ once every month) and antiandrogen (Bicalutamide $50 \mathrm{mg} / \mathrm{d}$ ). 
Table 1. Comparison of clinical data between 3 groups.

\begin{tabular}{llll}
\hline & Group A & Group B & Group C \\
\hline Number of cases (case) & 68 & 46 & 86 \\
Age (year) & $62.7(46 \sim 72)$ & $62.7(52 \sim 70)$ & $64.1(51 \sim 75)$ \\
PSA (ng/ml) & $11.0(3.4 \sim 33.3)$ & $11.7(5.6 \sim 17.8)$ & $12.9(5.0 \sim 34.7)$ \\
Clinical stages (case (\%)) & & & $50(58 \%)$ \\
cT1 & $54(79 \%)$ & $32(70 \%)$ & $36(42 \%)$ \\
cT2 & $14(21 \%)$ & $14(30 \%)$ & $42(48 \%)$ \\
The Gleason score of biopsy (case (\%)) & & & $34(40 \%)$ \\
$\leq 6$ & $32(47 \%)$ & $18(39 \%)$ & $10(12 \%)$ \\
7 & $32(47 \%)$ & $23(50 \%)$ & $44.3(18.4 \sim 121.9)$ \\
$\geq 8$ & $4(6 \%)$ & $5(11 \%)$ & $20(15 \sim 24)$ \\
\hline Prostate volume (cm2) & $44.7(21.2 \sim 86.4)$ & $48.1(32.1 \sim 64.1)$ & $19(15 \sim 25)$ \\
IIEF-5 score & $20(15 \sim 25)$ & $15)$ & \\
\hline
\end{tabular}

Notes: Pubovesical complex preserving technique for intrafascial laparoscopic radical prostatectomy (PPLRP) (group A), conventional intrafascial LRP (group B), interfascial LRP (group C). International Index of Erectile Function (IIEF-5).
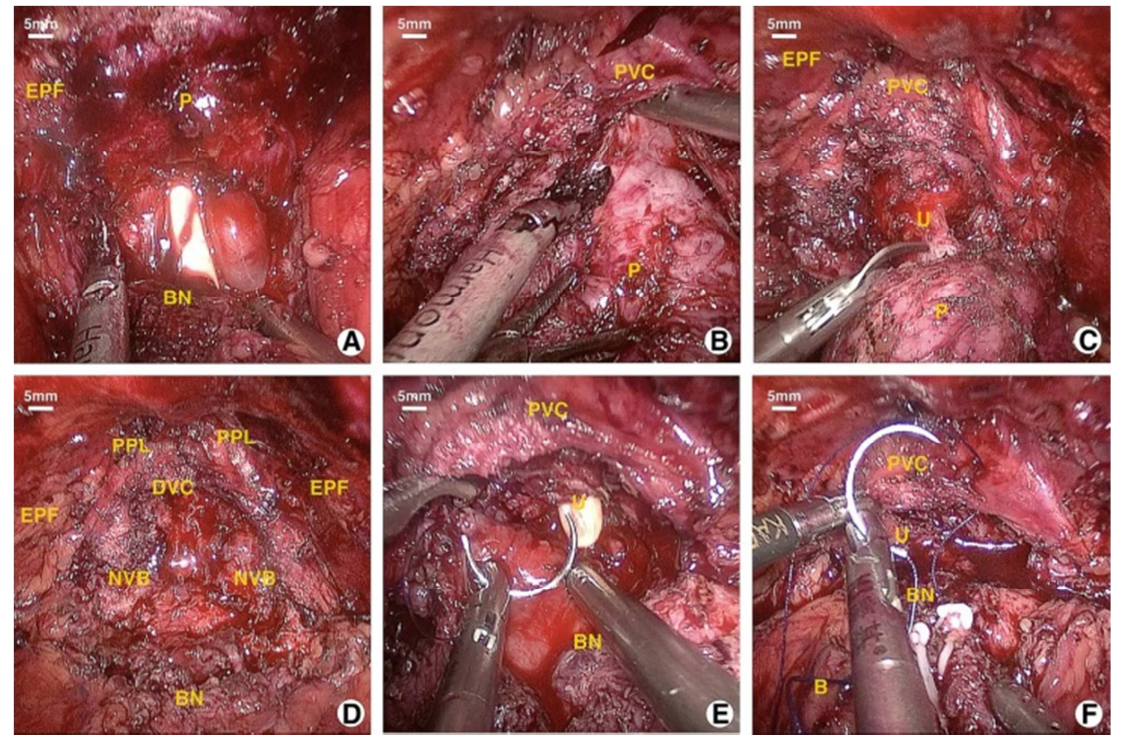

Figure 1. Pubovesical complex preserving technique for intrafascial laparoscopic radical prostatectomy (PPLRP) A. Preservation of the BN; B. Separation of prostate capsule and DVC; C. Cut the entire urethra horizontally with scissors; D. Completely preserved PVC, including DVC, detrusor apron, puboprostatic ligament and NVB; E. Reconstruct the posterior side of the BN, use the 2-0 absorbable suture, continuous suture bladder and urethra; F. Repairing PVC. (P, prostate; BN, bladder neck; EPF, endopelvina fascia; NVB, neurovascular bundle; PVC, pubovesical complex; PPL, pubic prostate ligament; DVC, dorsal vein complex; $U$, urethra; $B$, bladder).

Postoperative urinary continence functional was evaluated at immediately after catheter removal, 1,3 and 6 months after operation. Continence was defined as zero to one security pad per day. Sexual function was evaluated at 1, 3, and 6 months after operation using IIEF-5. PSA was checked every 3 months after operation, and if PSA has exceeded $0.2 \mathrm{ng} / \mathrm{mL}$ for two consecutive times, it suggests a biochemical recurrence of prostate cancer. Adjuvant radiotherapy or endocrine therapy was given after operation. Pelvic CT/MRI and SPECT/CT were performed to confirm the recurrence and metastasis of the tumor.

\subsection{Statistical Methods}

Data was processed using SPSS 16.0 statistical software. Enumeration data was checked by Chi-square test and Fisher exact test. Biochemical recurrence rate was checked by Kaplan-Meier Plot and Log-rank test. The difference was statistically significant with $\mathrm{P}<0.05$.

\section{Result}

\subsection{Perioperative Status}

Perioperative status of the three groups of patients are shown in Table 2. There was no significant difference in surgery time, intraoperative blood loss, number of transfusion cases, postoperative complications, and postoperative stay $(\mathrm{P}>0.05)$. There were 21 cases of bilateral nerve preservation in group A $(30.9 \%)$, and 14 cases in group B (30.4\%). The difference was not statistically significant $(\mathrm{P}>0.05)$. One patient in group $\mathrm{B}$ had postoperative anastomotic stenosis of the bladder and urethra and needed reoperation. One patient in group $\mathrm{C}$ developed lymphocytic cyst after operation. 
Table 2. Perioperative status of the three groups of patients.

\begin{tabular}{llll}
\hline & Group A & Group B & Group C \\
\hline Surgery time (min) & $195 \pm 68$ & $213 \pm 75$ & $191 \pm 72$ \\
Intraoperative blood loss (ml) & $282 \pm 94$ & $296 \pm 85$ & $277 \pm 72$ \\
Number of transfusion cases (case) & 2 & 2 & 1 \\
Postoperative complications (case) & 0 & 1 & 1 \\
Postoperative stay (day) & $8.3(6 \sim 17)$ & $8.1(6 \sim 15)$ & $8.5(5 \sim 16)$ \\
\hline
\end{tabular}

Note: Pubovesical complex preserving technique for intrafascial laparoscopic radical prostatectomy (PPLRP) (group A), conventional intrafascial LRP (group B), interfascial LRP (group C).

Table 3. Postoperative pathological results of the three groups of patients.

\begin{tabular}{|c|c|c|c|}
\hline & Group A & Group B & Group C \\
\hline \multicolumn{4}{|c|}{ Pathological staging (case (\%)) } \\
\hline pT2 & $59(87 \%)$ & $37(80 \%)$ & $67(78 \%)$ \\
\hline pT3 & $9(13 \%)$ & $9(20 \%)$ & $19(22 \%)$ \\
\hline \multicolumn{4}{|c|}{ Gleason score (case (\%)) } \\
\hline 6 & $31(46 \%)$ & $19(41 \%)$ & $33(39 \%)$ \\
\hline 7 & $30(44 \%)$ & $21(46 \%)$ & $39(45 \%)$ \\
\hline 8 & $7(10 \%)$ & $6(13 \%)$ & $14(16 \%)$ \\
\hline \multicolumn{4}{|c|}{ PSM rate (\% (case)) } \\
\hline pT2 & $5 \%(3 / 59)$ & $5 \%(2 / 37)$ & $6 \%(4 / 67)$ \\
\hline pT3 & $22 \%(2 / 9)$ & $44 \%(4 / 9)$ & $16 \%(3 / 19)$ \\
\hline \multicolumn{4}{|c|}{ PSM distribution (\% (case)) } \\
\hline Apex & $40 \%(2 / 5)$ & $50 \%(3 / 6)$ & $71 \%(5 / 7)$ \\
\hline Bilateral margins & $40 \%(2 / 5)$ & $16 \%(1 / 6)$ & 0 \\
\hline Bottom & $20 \%(1 / 5)$ & $33 \%(2 / 6)$ & $29 \%(2 / 7)$ \\
\hline
\end{tabular}

Note: Pubovesical complex preserving technique for intrafascial laparoscopic radical prostatectomy (PPLRP) (group A), conventional intrafascial LRP (group B), interfascial LRP (group C).

\subsection{Postoperative Pathological}

The results of pathological examination in 3 groups are shown in Table 3. All groups had similar PSM rate $(7.4 \%$ in group A, $13.0 \%$ in group B vs $8.1 \%$ in group C). And there was no significant difference in pathological staging and pathology, Gleason score, PSM rate and distribution $(\mathrm{P}>0.05)$.

\subsection{Postoperative Urinary Function}

Follow-up was more than 6 months, the postoperative urinary function of 200 patients who underwent LRP. Continence rates at immediately after catheter removal, 1,3 and 6 months: $68 \%(46 / 68), 85 \%(58 / 68), 93 \%(63 / 68)$ and $100 \%(68 / 68)$ in group A; and 63\% (29/46),80\% (37/46),89\% $(41 / 46)$ and $96 \%(44 / 46)$ in group B; and $22 \%(19 / 63), 49 \%$ (42/63),72\% (62/63) and 84\% (72/63) in group C. The group A showed a significantly earlier recovery from incontinence compared with that in the group $\mathrm{C}$ at immediately, 1,3 and 6 months after catheter removal, $\left(\chi^{2}=32.31, \mathrm{P}<0.01 ; \chi^{2}=21.17\right.$, $\left.\mathrm{P}<0.01 ; \chi^{2}=10.50, \mathrm{P}<0.01 ; \chi^{2}=10.29, \mathrm{P}<0.01\right)$; The group $\mathrm{B}$ showed a significantly earlier recovery from incontinence compared with that in the group $\mathrm{C}$ at immediately after catheter removal and 1 month, $\left(\chi^{2}=21.72, \mathrm{P}<0.01 ; \chi^{2}=12.45\right.$, $\mathrm{P}<0.01)$. There was no difference between the $\mathrm{A}$ and $\mathrm{B}$ groups at immediately after catheter removal and 1 month, $(\mathrm{P}$ $>0.25)$.

\subsection{Postoperative Erectile Function}

We followed up the postoperative erectile function of 200 patients who underwent LRP,Follow-up was more than 6 months. Regarding sexual function, at the postoperative 1,3,6 months, median IIEF-5 score was 10 (0 19), 11 (0 20), 16 $(0 \sim 24)$ in the group A, respectively; in the group B, it was 8 $(0 \sim 18), 9(0 \sim 19), 13(0 \sim 21)$ respectively; and in the group $\mathrm{C}$ it was $7(0 \sim 17), 8(0 \sim 19), 12(0 \sim 21)$ respectively. No differences were found in the three groups in potency (IIEF-5 score). At 6 months after operation, the percentage of erectile function recovered to preoperative state in group A, B and C was $50 \%(34 / 68), 35 \%(16 / 46)$ and $21 \%(18 / 86)$, respectively. There was a statistically significant difference between the group $\mathrm{A}$ and the group $\mathrm{C}\left(\chi^{2}=14.35, \mathrm{P}<0.01\right)$. There was no significant difference between the group $\mathrm{A}$ and the group $\mathrm{B}$ $\left(\chi^{2}=2.58, \mathrm{P}>0.1\right)$. There was no significant difference between group $\mathrm{B}$ and group $\mathrm{C}(\mathrm{X} 2=3.01, \mathrm{P}>0.05)$.

\subsection{Postoperative Survival}

We obtained data on the biochemical recurrence rate of 168 patients who underwent LRP. 59 patients (group A), 46 patients (group B) and 63 patients (group C). Follow-up was 31.6 (6-69) months without deaths. Biochemical relapse occurred in group A, B and C at 23, 21 and 13 months after operation, respectively, with 6,9 , and 18 cases, respectively. Biochemical recurrence-free survival at 3 years was $79.3 \%, 76.3 \%$ and $76.4 \%$ of $\mathrm{A}, \mathrm{B}$ and $\mathrm{C}$ group, respectively. The difference between three groups was not significant $\left(\chi^{2}\right.$ $=0.405, \mathrm{P}>0.05$, Figure 2). Pelvic CT/MRI and SPECT/CT were performed to confirm the recurrence and metastasis of the tumor. No metastases were found in group A. One patient in group B had recurrence of bone metastases. In group C, 
there was one patient of bone metastases, and one patient had pelvic recurrence with pulmonary metastasis. There were 1,1 and 3 patients respectively in groups $\mathrm{A}, \mathrm{B}$, and $\mathrm{C}$ receiving salvage radiotherapy, respectively, using intensity-modulated radiation therapy: Total external radiation dose was $40 \mathrm{~Gy}$, 2.0 Gy qd, 5 days per week.

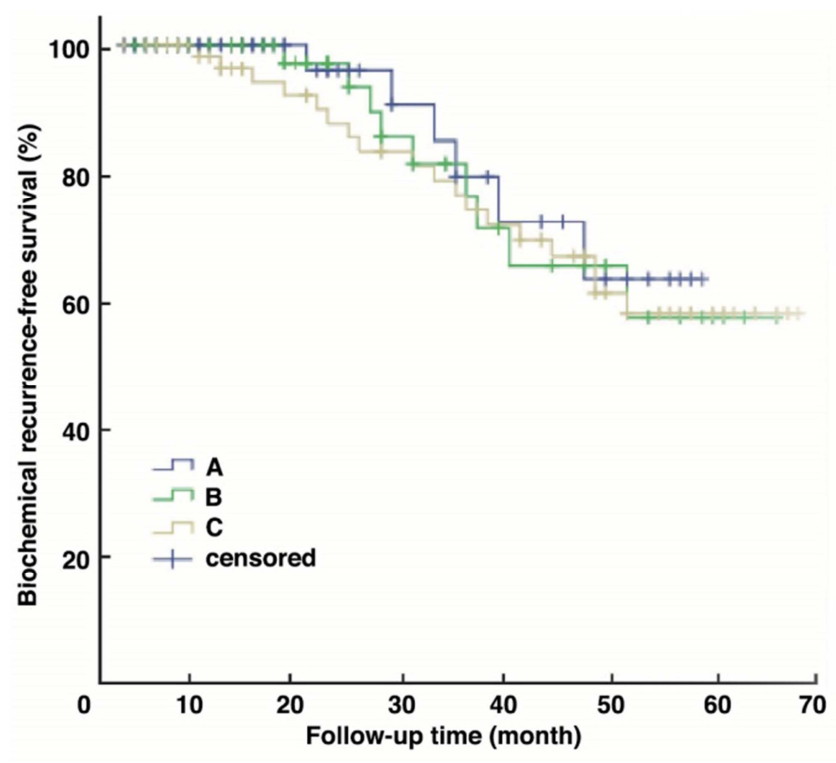

Figure 2. Comparison of postoperative biochemical recurrence-free survival of the three groups of patients undergoing LRP Note: pubovesical complex preserving technique for intrafascial laparoscopic radical prostatectomy (PPLRP) (group A), conventional intrafascial LRP (group B), interfascial LRP (group $C$ ).

\section{Discussion}

Doctors and patients hope that when the catheter was removed after the operation, the urinary control function and the sexual function could be restored as soon as possible [6, 7]. Therefore, more scholars have conducted in-depth anatomical studies to accurately dissect the nerves and blood vessels around the prostate and urethra [8, 9]. PPLRP technology is an improvement based on the "The veil of Aphrodite technique" [10], which retains NVB extensively in robotic assisted radical prostatectomy (RARP).

There are some advantages of PPLRP technology: Firstly, this technology maximizes the retention, reconstruction of anatomy around the prostate, including puboprostatic ligament, detrusor apron, DVC, NVB, accessory pudendal artery and BN. Compared with conventional intrafascial LRP, the prostate is dissected under DVC, which can completely preserve DVC, avoid damaging the sphincter around the urethra when sewing DVC. Like perineal radical prostatectomy, where the prostate is dissected from the detrusor apron and under DVC[11], PPLRP technology can completely preserve the anatomical support structure of the external urinary sphincter, allowing the urethra can be placed in the normal anatomy position of the pelvic floor and stable without additional additions operations such as hanging the urethral complex[12,13] and Pubic perineoplasty [14]. At the same time, this technology repairs and reconstructs the tissue structure such as urinary control around the urethra and BN. Secondly, this technology contributes to earlier recovery from incontinence. The literature reports that the continence rates were $61.9 \%$ and $90.5 \%$ at 1 month and 3 months after neuro-preservation RARP, respectively [15], and the continence rates at 3 months after RARP with $\mathrm{BN}$ preservation technique were $84.2 \%$ [16]. The results of this study showed that the continence rates at 1 and 3 months after PPLRP were $85 \%$ and $93 \%$, respectively. The data in this study is higher than those reported in the literature, which may be related to the complete retention of $\mathrm{BN}$ and PVC. In this study, the group A showed a significantly earlier recovery from incontinence compared with that in the group B (at immediately after catheter removal and 1month), compared with group $\mathrm{C}$, the group A showed a significantly earlier recovery from incontinence at immediately, 1, 3 and 6 months after catheter removal, indicating that complete retention of $\mathrm{BN}$ and $\mathrm{PVC}$ can restore postoperative urinary function earlier. Thirdly, this technique can restore postoperative sexual function relatively quickly. The literature reports that the Erectile function recovery rate was $38.4 \%$ at 3 months after RARP [17]. The results of this study show that 3 groups of postoperative there was no statistically significant difference in the IIEF-5 scores at 1, 3, and 6 months. The proportion of patients with IIEF-5 scores restored to preoperative level in group A was higher than that in group $\mathrm{C}$ at 6 months after operation, but there was no difference between group A and group B. Finally, this technology has a reliable effect. The literature reports that biochemical recurrence-free survival at 3 years was $60.4 \% \sim 76.0 \%[18,19]$ after LRP. The results of this study show that 3 groups of postoperative there was no statistically significant difference in biochemical recurrence-free survival at 3 years, of which group A was $79.3 \%$, similar to the literature report, indicating that the short-term efficacy of PPLRP is effective, and its long-term efficacy still needs further follow-up study.

PPLRP may increase the PSM rate due to the extensive preservation of nerves and blood vessels around the prostate. The results of this study show that the PSM rates of the pT2 and pT3 in group A were similar to those in the other 2 groups, indicating that the PPLRP did not increase the PSM rate, especially in stage pT2. The literature reports that the PSM rate in traditional surgery of the pT2 was $4.5 \% \sim 17.5 \%$ $[2,20,21]$. In this study, the PSM rate of PT2 in group A was $5 \%$, similar to that in traditional surgery. The most common anatomical site of PSM in PPLRP is the two sides and the tip of the prostate. Therefore, for patients using PPLRP, MRI examination must be performed before surgery, and biopsies of both sides of the prostate and the tip of the prostate are required. so as to identify the location of the tumor in the prostate, and to select or not to select PPLRP based on the anatomy of the tumor. When a suspicious tumor capsule infiltration is found during surgery, we should undergo intraoperative rapid freezing section for pathological examination, if a positive margin is found, the extent of resection should be expanded. 
Through this study, we conclude that the indication of adopting PPLRP is prostate cancer of $\leq \mathrm{T}_{2}$. PPLRP is not recommended when the tumor is located on the side of the prostate capsule and on the ventral side of the tip of the prostate, or prostate cancer of $\geq T_{3}$. A more extensive surgical procedure, such as interfascial LRP, should be used.

There are some precautions for using PPLRP: Firstly, medium-sized prostate surgery should be selected at the beginning, and the surgical technique should be mastered before expanding the surgical indications. PPLRP retains $\mathrm{PVC}$, and it is difficult to find and dissect the vascularless plane between PVC and prostatic urethra. For small volume prostate, the vascularless plane between PVC and prostatic urethra is extremely short, and it is easy to damage the sphincter urethrae, NVB and DVC. For large volume prostate, detrusor apron and DVC due to extrusion thinning and dispersing, it is difficult to separate the anatomy from the front. Secondly, due to the retention of PVC, the vision is narrow in the course of anastomosis. Use a non-invasive grasping forceps lifting the detrusor apron, exposing the junction of the prostatic urethra, is conducive to anastomosis. Finally, Because of the extensive retention of NVB, intraoperative bleeding is more than traditional intrafascial LRP, during operation, small vessels can be clipped by titanium clips or Hem-o-lok.

However, the study has some shortcomings. Firstly, this is a retrospective study with a small sample size, possible bias in data, requiring randomized control and more sample studies. Secondly, the treatments for patients with PSM and non-metastatic biochemical recurrence are inadequate. Guidelines recommend the first recommended treatment for patients with PSM and non-metastatic recurrence in recent years are local adjuvant radiotherapy and salvage radiotherapy for prostatic bed (or endocrine therapy) [22]. This study mainly used endocrine therapy, and only some patients performed salvage radiotherapy. Although the short-term effects are acceptable, the long-term effects are still unclear. Therefore, patients should be treated strictly according to the guidelines.

\section{Conclusions}

The PVC preserving technique for LRP provides early recovery from incontinence, faster recovery of sexual function preoperative levels. There is no adverse effect on the oncological outcome in the selective prostate cancer. It is an ideal way of nerve-sparing LRP.

\section{Acknowledgements}

To acknowledge all of the people who have contributed to this paper. And my deepest gratitude foremost to Professor Zaisheng Zhu, my supervisor, for his constant encouragement and guidance. He has walked me through all the stages of the writing of this thesis. Without his consistent and illuminating instruction, this thesis could not have reached its present form.

\section{References}

[1] Haga N, Ogawa S, Yabe M, et al. Factors Contributing to early recovery of urinary continence analyzed by pre- and postoperative pelvic anatomical features at robot-assisted laparoscopic radical prostatectomy [J]. J Endourol, 2015, 29: 683-690. DOI: 10. 1089 / end. 2014. 0708.

[2] Laird A, Fowler S, Good DW, et al. Contemporary practice and technique-related outcomes for radical prostatectomy in the UK: a report of national outcomes [J]. BJU Int, 2015, 115: 753-763. DOI: 10. 1111 / bju. 12866.

[3] Al-Mamari SA, Quintens H, Mentine N, et al. RALP: comparison of the oncological and functional outcomes of the intrafascial and the interfascial approaches [J]. Prog Urol, 2015, 25: 54-61. DOI: 10. 1016 / j. purol. 2014. 08. 237.

[4] Zaisheng Zhu, Qiang Fu, Lizhen $\mathrm{Xu}$, et al. Effect of the modified nerve-sparing technique in open antegrade radical prostatectomy for 30 patients with prostate cancer [J]. Cancer Research and Clinic, 2015, 27: 680-686. DOI: 10. 3760 / cma. j. issn. 1006-9801. 2015. 10. 008.

[5] Riikonen J, Kaipia A, Petas A, et al. Initiation of robot-assisted radical prostatectomies in Finland: impact on centralization and quality of care [J]. Scand J Urol, 2016, 53: 149-154. DOI: 10.3109/21681805. 2016. 1142471.

[6] Yanqun Na, Yinghao Sun. Guidelines for the diagnosis and treatment of prostate cancer [M] / / Yanqun Na, Zhangqun Ye, Yinghao Sun, et al. The 2014 edition of Chinese Department of Urology guidelines for disease diagnosis and treatment. Beijing: People's Medical Publishing House, 2014: 61-89.

[7] Papachristos A, Basto M, Te Marvelde L, et al. Laparoscopic versus robotic-assisted radical prostatectomy: an Australian single-surgeon series [J]. ANZ J Surg, 2015, 85: 154-158. DOI 10.1111 / ans. 12602 .

[8] Tran SN, Wirth GJ, Mayor G, et al. Prospective evaluation of early postoperative male and female sexual function after radical prostatectomy with erectile nerves preservation $[\mathrm{J}]$. Int J Impot

[9] Res, 2015, 27: 69-74. DOI: 10. 1038 / ijir. 2014. 36. [9] Walz J, Burnett AL, Costello AJ, et al. A critical analysis of the current knowledge of surgical anatomy related to optimization of cancer control and preservation of continence and erection in candidates for radical prostatectomy [J]. Eur Urol, 2010, 57: 179-192. DOI: 10. 1016/ j. eururo. 2009. 11. 009.

[10] Tunc L, Akin Y, Gumustas H, et al. Detailed surgical anatomy of prostate: relationship between urethra and dorsal vein complex with apex [J]. Urol Int, 2016, 96: 260-267. DOI: 10. $1159 / 000443674$.

[11] Lim SK, Kim KH, Shin TY, et al. Retzius-sparing robot-assisted laparoscopic radical prostatectomy: combining the best of retropubic and perineal approaches [J]. BJU Int, 2014, 114: 236-244. DOI:10. 1111 / bju. 12705.

[12] Walz J, Epstein JI, Ganzer R, et al. A critical analysis of the current knowledge of surgical anatomy of the prostate related to optimisation of cancer control and preservation of continence and erection in candidates for radical prostatectomy: an update [J] Eur Urol, 2016, 70: 301-311. DOI: $10.1016 /$ j. eururo. 2016. 01. 026. 
[13] Papatsoris A, Mandron E. Anrerior suspension of the dorsal vein complex and fixation of the anterior fibromuscular stroma during laparoscopic prostatectomy for facilitating early continence [J]. BJU Int, 2009, 104: 1542-1546. DOI: 10 1111/ j. 1464-410X. 2009. 09009. X.

[14] Takenaka A, Tewari AK, Leung RA, et al. Preservation of the puboprostatic collar and puboperineoplasty for early recovery urinary continence after robotic prostatectomy anatomic basis and preliminary outcomes [J]. Eur Urol, 2007, 51: 433-440. DOI: $10.1016 /$ j. eururo. 2006. 07. 007.

[15] Zhenkai Shi, Xu Gao, Haifeng Wang, et al. Effect of robot assisted radical prostatectomy on postoperative urinary continence [J / CD]. Chinese Journal of endourology (electronic version), 2016, 10: 9-12. DOI: 10. 3877 / cma. j. issn. 1674-3653. 2016. 01. 003.

[16] Xinjian Liu, Jianguo Wen, Qingwei Wang, et al. Meta-analysis of bladder neck preservation during radical prostatectomy for postoperative urinary function recovery $[\mathrm{J}]$. Chinese Journal of Urology, 2016, 37: 174-178. DOI: 10.3760 / cma. j. issn. 1000-6702. 2016. 03. 004.

[17] Alemozaffar M, Sanda M, Yecies D, et al. Benchmarks for operative outcomes of robotic and open radical prostatectomy: results from the Health Professionals Follow-up Study [J]. Eur Urol, 2015, 67: 432-438. DOI: 10. 1016 / j. eururo. 2014. 01. 039 .
[18] Xun Shangguan, Baijun Dong, Jiayu Pan, et al. Establishment and evaluation of nomogram for predicting biochemical recurrence in patients with locally adverse pathologic features after radical prostatectomy [J]. Chinese Journal of Urology, 2016, 37: 174-178. DOI: 10. 3760 / cma. j. issn. 1000-6702. 2016. 03.004.

[19] Zhiyuan Shen, Weiqing Qian, Wei Sheng, et al. Comparative study on the efficacy and safety of robot assisted laparoscopic and retropubic radical prostatectomy [J]. Chinese Journal of Urology, 2015, 36: 174-178. DOI: $10.3760 / \mathrm{cma}$. j. issn. 1000-6702. 2016. 08.010.

[20] Busch J, Gonzalgo ML, Leva N, et al. Matched comparison of robot-assisted, laparoscopic and open radical prostatectomy regarding pathologic and oncologic outcomes in obese patients [J]. World J Urol, 2015, 33: 397-402 DOI: 10. 1007 / s00345-014-1326-1.

[21] Soares R, Di Benedetto A, Dovey Z, et al. Minimum 5-year follow up of 1138 consecutive laparoscopic radical prostatectomies [J]. BJU Int, 2015, 115: 546-553. DOI: 10.1111/bju.12887.

[22] Fang Guo, Zhao Liu, Xiaodong Xie. Interpretation and discussion of update points for NCCN prostate cancer clinical treatment guideline [J], Chinese Journal of Urology, 2013, 34: 949-951. DOI: 10. 3760 / cma. j. issn 1000-6702. 2013. 12. 020. 